\title{
Regulation of Notch Signaling by Glycosylation
}

\author{
Pamela Stanley, PhD \\ Dept. Cell Biology, Albert Einstein College of Medicine, 1300 Morris Park Ave., New York, NY, \\ 10461, email: stanley@aecom.yu.edu, Phone: 1-718-430-3346, Fax: 1-718-430-8574
}

\section{Summary}

Notch receptors are $\sim 300 \mathrm{kd}$ cell surface glycoproteins whose activation by Notch ligands regulates cell fate decisions in the metazoa. The extracellular domain of Notch receptors has many epidermal growth factor-like repeats that are glycosylated with O-fucose and O-glucose glycans as well as Nglycans. Disruption of O-fucose glycan synthesis leads to severe Notch signaling defects in Drosophila and mammals. Removal or addition of O-fucose glycan consensus sites on Notch receptors also leads to Notch signaling defects. Ligand binding and ligand-induced Notch signaling assays have provided insights into how changes in the O-fucose glycans of Notch receptors alter Notch signaling.

\section{Introduction}

Notch signaling controls the fate of many cell types in the metazoa and dysregulation of Notch signaling leads to developmental defects or cancer [1]. In the canonical Notch signaling pathway the Notch ligands Delta or Serrate (in Drosophila) and Delta-like or Jagged (in mammals) bind to the extracellular domain of Notch receptors (NECD) on apposing cells and two sequential proteolytic cleavages ensue. The first, caused by an ADAM (disintegrin and metalloprotease), occurs close to the Notch transmembrane region on the outside of the cell. The second occurs within the transmembrane domain of Notch and is induced by a complex of proteins that include presenilins with $\gamma$-secretase activity. The released Notch intracellular domain (NICD) complexes with the transcriptional repressor CSL (CBF-1/Suppressor-ofhairless/Lag-1), recruits the co-activator Mastermind (Maml) and activates Notch target genes, including transcriptional regulators such as Hes and Hey genes. Drosophila contains one Notch receptor and two Notch ligands whereas mammals have four Notch receptors and five Notch ligands (three Delta and two Jagged).

Interest in the roles of glycans in Notch signaling began with the discovery that the Drosophila Fringe gene (FNG), a known regulator of Notch signaling, encodes a glycosyltransferase which transfers $\mathrm{N}$-acetylglucosamine (GlcNAc) to fucose on Notch epidermal growth factor-like (EGF) repeats [2,3] (Fig. 1). Fringe may modify other proteins that contain a Fuc-O-EGF domain, including Notch ligands [4]. The EGF repeats of mammalian Notch1 produced in cultured cells carry O-fucose glycans, O-glucose glycans and complex N-glycans (Fig. 2). All Notch receptors have consensus sequences known to be modified by these glycans. Mutant mice lacking complex N-glycans, or core 1-derived mucin O-glycans, or numerous other sugars are not defective in Notch signaling during embryonic

Correspondence to: Pamela Stanley.

Publisher's Disclaimer: This is a PDF file of an unedited manuscript that has been accepted for publication. As a service to our customers we are providing this early version of the manuscript. The manuscript will undergo copyediting, typesetting, and review of the resulting proof before it is published in its final citable form. Please note that during the production process errors may be discovered which could affect the content, and all legal disclaimers that apply to the journal pertain. 
development $[5,6]$. However, inactivation of glycosyltransferases or other activities required for O-fucose glycan synthesis gives rise to Notch signaling defects in Drosophila [7], zebrafish [8], sea urchin [9] and mammals [10] (Table 1). Glycosyltransferases involved in glycolipid synthesis are also necessary for Notch signaling [11], an indirect effect that probably has to do with the appropriate organization of Notch receptors in membrane domains. This review will focus on recent mechanistic insights into how the O-fucose glycans of Drosophila and mammalian Notch receptors are proposed to regulate Notch signaling.

\section{Notch receptors lacking O-fucose glycans}

Notch receptors lacking O-fucose are inactive and the question is why? To determine if Notch devoid of O-fucose binds to Notch ligands, soluble Dros. Notch ECD linked to alkaline phosphatase (N-AP) produced from S2 cells in the presence and absence of OFUT1 was investigated for binding to $\mathrm{S} 2$ cells expressing Delta or Serrate [12]. N-AP from control cells binds well to both ligands, though binding is not saturable. N-AP from S2 cells lacking OFUT1 did not bind to Delta/S2 or Serrate/S2 cells, consistent with a role for O-fucose in ligand recognition. However, N-AP is secreted poorly in the absence of OFUT1. Drosophila S2 cells have an appreciable level of endogenous OFUT1 and efficiently secrete N-AP that binds Notch ligands. However, overexpression of OFUT1 produces N-AP with enhanced ligand binding activity without apparently increasing its $\mathrm{O}$-fucose content. This result indicated a fucosetransfer-independent activity of OFUT1.

Two groups have now shown that Dros. OFUT1 binds directly to Notch when both Notch and OFUT1 are overexpressed in S2 cells [13,14]. Each group proposes that OFUT1 is necessary for the stable cell surface expression of Notch receptors. However, there are three proposed mechanisms for how OFUT1 functions. Okajima et al. [13] provide evidence that Notch is trapped in the endoplasmic reticulum (ER) in the absence of OFUT1. A mouse mutant Pofut1 that lacks fucosyltransferase activity, Pofut1 R245A, enhances the secretion and ligand binding ability of soluble N-AP from S2 cells lacking OFUT1 [13]. In addition, transgenic expression

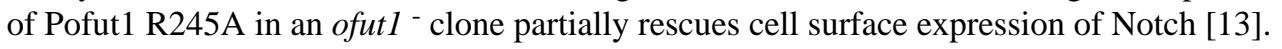
Sasamura et al. [14] use deconvoluted confocal micrographs to conclude that Notch does not completely colocalize with conventional ER markers in Drosophila cells lacking OFUT1. By tracking Notch in live cells in real time, Sasamura et al [14] find that Notch is expressed equivalently at early times on the surface of ofut $1^{-}$and wild type cells, but is rapidly endocytosed in the absence of OFUT1 and accumulates in a novel endocytic compartment. Exogenously added OFUT1 reduces Notch accumulation and the authors suggest that secreted OFUT1 bound to cell surface Notch regulates constitutive endocytosis of Notch receptors [14]. In a related paper, however, the same authors conclude that OFUT1 is necessary for transcytosis of Notch at the cell surface to the subapical complex of the adherens junctions [15]. Clearly, technical issues regarding the basis for an antibody to accumulate in a particular compartment in a live cell, and the resolving power of confocal images for localization to intracellular compartments of small Drosophila cells, need to be further investigated. Most critical is to determine whether Notch receptors made in ofut $1^{-}$cells expressing mouse Pofut1 R245A at temporally regulated, physiological levels, may signal upon encountering Notch ligands.

\section{Notch receptors in the absence of Fringe}

In the Drosophila wing disc Fringe enhances Notch signaling induced by Delta and inhibits Notch signaling induced by Serrate to restrict Notch signaling to a stripe of cells at the dorsal/ ventral boundary [7]. Notch receptors made in the absence of Fringe are predicted to bear Ofucose at 23 of 36 EGF repeats in Dros. Notch. The action of Fringe alters Notch ligand binding. Thus soluble Dros. N-AP co-expressed with Fringe in S2 cells binds much better to Delta/S2 
cells and significantly worse to Serrate/S2 cells compared to N-AP containing O-fucose but not modified by Fringe $[12,16]$. However, to investigate roles for sugars in O-fucose glycans directly, it will be important to develop cell-free, in vitro binding assays with purified Notch and ligand ECDs bearing structurally-characterized glycans. Even so, such assays may not appropriately reflect in vivo interactions between Notch and ligands which occur in specialized, dynamic, plasma membrane compartments of apposing cells. In this regard, atomic force microscopy may provide the most physiologically relevant data [17].

Overexpression of mammalian Fringe genes in cultured cells alters Notch signaling levels. In general, Lunatic Fringe (Lfng) and Manic Fringe (Mfng) inhibit Jagged1-induced Notch signaling and increase Delta1-induced Notch signaling. However, differences between the effects of Lfng and Mfng on Notch2 signaling have been observed [18], and Radical Fringe (Rfng) overexpression in NIH3T3 cells enhances both Delta1- and Jagged1-induced Notch signaling [19]. Lfng, Mfng and Rfng differ in in vitro specific activity [20], and their activities may not be equivalent in different cell types. The same concerns apply to defining the effects of Fringe on ligand binding. In general, the binding of soluble Delta1 ECD to Notch1 or Notch2 assayed by flow cytometry or immunofluorescence microscopy has conformed to predictions [19,21]. By contrast, Delta3 does not bind to Notch1 nor activate Notch signaling [22]. In addition, Jagged1 binding is not significantly altered by overexpression of Lfng, Mfng or Rfng, despite the fact that Jagged1-induced Notch1 signaling is affected by each Fringe in signaling assays [19]. This finding led to the proposal that O-fucose modified by Fringe may primarily affect the strength of Notch-ligand binding rather than recognition of Notch by ligands [19]. Elegant assays that follow the endocytosis of Notch1 into Delta1- or Jagged1-expressing cells [21] suggest that Notch endocytosis into the ligand-expressing cell, long known to be essential for Notch signaling to be initiated, occurs before the ADAM cleavage (Fig. 1) rather than afterwards, as previously believed. It will be of great interest to perform these endocytosis assays in cells expressing Notch in the presence and absence of the three mammalian Fringe enzymes. Of concern in transfection assays in cultured cells however, are effects of overexpressed Fringe that potentially modify Notch EGF repeats which are not normally substrates for Fringe under physiological conditions. In addition, there may be activities in transfected cells that further modify Notch EGF repeats after they have been acted on by Fringe. For example, in $\mathrm{CHO}$ cells the action of Fringe is followed by elongation of the $\operatorname{GlcNA} \beta(1,3)$ Fuc-O disaccharide by a Gal residue to which a sialic acid may be added to give sialic acid $\alpha$ $(2,3) \mathrm{Gal} \beta(1,4) \mathrm{GlcNAc} \beta(1,3)$ Fuc-O [23]. Indeed Lfng and Mfng were found to be necessary but not sufficient for a Fringe effect in a $\mathrm{CHO}$ co-culture signaling assay [24]. The addition of Gal was required to obtain modulation of Notch signaling by Fringe. Gal has been found in Nlinked glycans of Drosophila embryos [25] and therefore a Gal-transferase that may potentially add Gal to O-fucose glycans is expected to exist in Drosophila. In mice lacking $\beta 4 G a l T-1$, several Notch target genes involved in somitogenesis are poorly expressed at mid-gestation, and the majority of late term embryos have an extra lumbar vertebra [26]. Mice express five additional $\beta 4$ GalTs that may ameliorate a defective Notch signaling phenotype when only $\beta 4$ GalT-1 is absent.

\section{Elimination or Acquisition of O-Fucose Sites in Notch}

In CHO cells, Fringe modifies O-fucose on many, but not all, Notch1 EGF repeats in Notch1 $\mathrm{ECD}$ fragments that have the consensus O-fucose site $\mathrm{C}^{2} \mathrm{X}_{4-5} \mathrm{~T} / \mathrm{SC}^{3}$ [27]. Thus mutation of an $\mathrm{O}$-fucose consensus site eliminates not only the transfer of O-fucose to Notch but also the effects of Fringe at that site. One of the most conserved O-fucose sites on all Notch receptors, including those of C. elegans, is in EGF 12 [28]. Deletion experiments have confirmed that EGF 11 and 12 constitute the Notch ligand binding domain [28]. While Notch1 EGF repeats 11-13 made in E.coli, and therefore not O-fucosylated, bind to Delta-expressing cells, this was only observed after tetramerization of the Notch fragment [29]. In fact, elimination of O-fucose 
from EGF 12 has profound conequences for Notch signaling. In Drosophila, conversion of Thr to Ala in Notch EGF 12 generates Notch that is hyperactive in response to ligands when ectopically expressed, leading to the conclusion that the O-fucose glycan at EGF 12 suppresses Notch activity [16]. Binding experiments performed with Dros. N-AP produced in S2 cells expressing different levels of OFUT1 or Fringe show that N-AP lacking EGF 11 and 12 has no ligand binding activity [28]. However, NAP with a point mutation eliminating only the Ofucose site in EGF 12 (N-AP 12f) binds well to Delta/S2 and Serrate/S2 cells. In fact, ligand binding is greater for N-AP $12 \mathrm{f}$ than N-AP, consistent with the evidence that this O-fucose site suppresses Notch signaling in Drosophila [16]. In addition, binding to Delta/S2 cels is increased and to Serrate/S2 cells is decreased for N-AP $12 \mathrm{f}$ modified by Fringe, albeit the respective fold-increase or -decrease is not as great as obtained for control N-AP. Removal of O-fucose from Dros. Notch EGF repeats 24, 26, 24 and 26 or 31 has no effect in an ectopic Notch signaling assay [16]. In vitro binding assays showed a less than $50 \%$ decrease in binding of Delta or Serrate to N-AP lacking O-fucose in EGF repeats 23 to 32, but no effect on Fringe enhancement of Delta1 binding or inhibition of Serrate binding [28].

In stark contrast and surprisingly, the $12 \mathrm{f}$ mutation in mammalian Notch1 has the opposite effect and causes inhibition of Notch signaling induced by Delta1 or Jagged 1 [30,31]. Therefore the O-fucose in EGF 12 in the ligand binding domain of Notch1 is important for positive ligand interactions. O-fucose itself appears to be the key since replacement of the Thr in Notch1 EGF 12 with Ser which acccepts fucose, does not affect Notch1 signaling [31]. Removal of other conserved O-fucose sites in Notch1 at EGF 26 and 27 also has consequences. In CHO cells, Notch1 lacking O-fucose at EGF 26 responds better to both Delta1/L and Jagged1/L cells whereas removal of O-fucose from EGF 27 inhibits signaling induced by both ligands [30]. In the latter case, the basis for reduced signaling appears to be a reduction in Notch processing by furin protease in the Golgi. Therefore, removal of O-fucose from an EGF repeat gives rise to various consequences that are not predictable, and apparently not conserved between Drosophila and mammals. It will be important to replace the Ser or Thr that normally carries fucose with Thr or Ser, respectively, to be sure that loss of the fucose and not the amino acid change is the basis of an observed phenotype [31]. In addition, experiments to determine Ofucose functions should be performed in vivo with Notch mutants expressed at physiological levels from the endogenous locus.

There is one example of a gain-of-function phenotype due to the acquisition of O-fucose in Dros. Notch EGF 14 which has no O-fucose consensus site [32]. In the Notch split mutation, a Thr is introduced into EGF 14 and becomes O-fucosylated. This causes neuronal precursor cells to be stimulated by R8 Delta-containing cells in the ommatidium of the eye, to differentiate and then die. This phenotype does not depend on Fringe which suggests that the acquisition of a single O-fucose in EGF 14 may cause Notch in neuronal precursor cells to be inappropriately activated by Delta1 in R8 cells.

\section{Conclusions}

The O-fucose glycans of Notch receptors are important modulators of Notch signaling. Complete removal by mutating OFUT1/Pofut1 results in severe, global Notch signaling defects. In Drosophila this appears to be due in part to the loss of chaperone effects of OFUT1. However, O-fucose itself as well as Fringe, are necessary for boundary formations in the wing disc, eyes and legs. Binding of Dros. Notch ECD to ligand-expressing cells shows that Fringe causes increased binding of Delta and decreased binding of Serrate. Some variations on this theme are observed in mammalian cells that have several non-redundant Notch receptors, Notch ligands and Fringes. However, in vitro assays are fraught with potential problems. They may not reflect binding interactions that occur in membrane-anchored, potentially oligomeric Notch receptors and ligands, highly organized in a cell membrane and poised to be endocytosed. 
Thus, it is of key importance to develop methods to determine the precise structure of each Ofucose glycan on Notch isolated from specialized cell types in vivo. Steps in this direction have been initiated [33] but the sensitivity of mass spectroscopic techniques is currently not up to the task of analysing site-specific glycosylation in small numbers of cells such as might be obtained by laser capture microscopy.

\section{Acknowledgements}

The author acknowledges the many contributions to this field for which original references could not be given due to space limitations. This work was supported by National Institutes of Health grant RO1 CA 95022 to P. S.

\section{References}

1. Bolos V, Grego-Bessa J, de la Pompa JL. Notch signaling in development and cancer. Endocr Rev 2007;28:339-363. [PubMed: 17409286]

2**. Moloney DJ, Panin VM, Johnston SH, Chen J, Shao L, Wilson R, Wang Y, Stanley P, Irvine KD, Haltiwanger RS, et al. Fringe is a glycosyltransferase that modifies Notch. Nature 2000;406:369375. [PubMed: 10935626] These two papers show that the activity encoded in the Fringe gene, shown originally in Drosophila to modulate Notch signaling, is a GlcNAc-transferase in Drosophila and mammals.

3**. Bruckner K, Perez L, Clausen H, Cohen S. Glycosyltransferase activity of Fringe modulates NotchDelta interactions. Nature 2000;406:411-415. [PubMed: 10935637] These two papers show that the activity encoded in the Fringe gene, shown originally in Drosophila to modulate Notch signaling, is a GlcNAc-transferase in Drosophila and mammals.

4. Panin VM, Shao L, Lei L, Moloney DJ, Irvine KD, Haltiwanger RS. Notch ligands are substrates for protein O-fucosyltransferase-1 and Fringe. J Biol Chem 2002;277:29945-29952. [PubMed: 12036964]

5. Haltiwanger RS, Lowe JB. Role of glycosylation in development. Annu Rev Biochem 2004;73:491537. [PubMed: 15189151]

6. Xia L, Ju T, Westmuckett A, An G, Ivanciu L, McDaniel JM, Lupu F, Cummings RD, McEver RP. Defective angiogenesis and fatal embryonic hemorrhage in mice lacking core 1-derived O-glycans. J Cell Biol 2004;164:451-459. [PubMed: 14745002]

7. Haines N, Irvine KD. Glycosylation regulates Notch signalling. Nat Rev Mol Cell Biol 2003;4:786797. [PubMed: 14570055]

8. Appel B, Marasco P, McClung LE, Latimer AJ. lunatic fringe regulates Delta-Notch induction of hypochord in zebrafish. Dev Dyn 2003;228:281-286. [PubMed: 14518000]

9. Peterson RE, McClay DR. A Fringe-modified Notch signal affects specification of mesoderm and endoderm in the sea urchin embryo. Dev Biol 2005;282:126-137. [PubMed: 15936334]

10. Lu L, Stanley P. Roles of O-fucose glycans in notch signaling revealed by mutant mice. Methods Enzymol 2006;417:127-136. [PubMed: 17132502]

11*. Chen YW, Pedersen JW, Wandall HH, Levery SB, Pizette S, Clausen H, Cohen SM. Glycosphingolipids with extended sugar chain have specialized functions in development and behavior of Drosophila. Dev Biol 2007;306:736-749. [PubMed: 17498683] Functions of Drosophila glycolipids synthesized by Brainiac and Egghead that affect EGF receptor and Notch signaling.

12*. Okajima T, Xu A, Irvine KD. Modulation of notch-ligand binding by protein O-fucosyltransferase 1 and fringe. J Biol Chem 2003;278:42340-42345. [PubMed: 12909620] In vitro binding data show that the action of Fringe enhances the binding of Dros. Notch ECD to Delta and inhibits binding to Serrate.

13**. Okajima T, Xu A, Lei L, Irvine KD. Chaperone Activity of Protein O-Fucosyltransferase 1 Promotes Notch Receptor Folding. Science 2005;307:1599-1603. [PubMed: 15692013] First evidence that OFUT1/Pofut1 functions in the ER and has a chaperone activity independent of its fucosyltransferase activity.

14**. Sasamura T, Ishikawa HO, Sasaki N, Higashi S, Kanai M, Nakao S, Ayukawa T, Aigaki T, Noda $\mathrm{K}$, Miyoshi E, et al. The O-fucosyltransferase O-fut1 is an extracellular component that is essential 
for the constitutive endocytic trafficking of Notch in Drosophila. Development 2007;134:13471356. [PubMed: 17329366] Confocal microscopic evidence that, in the absence of OFUT1, Dros. Notch does not accumulate in the conventional ER but in a novel endocytic compartment.

15. Sasaki N, Sasamura T, Ishikawa HO, Kanai M, Ueda R, Saigo K, Matsuno K. Polarized exocytosis and transcytosis of Notch during its apical localization in Drosophila epithelial cells. Genes Cells 2007;12:89-103. [PubMed: 17212657]

16. Lei L, Xu A, Panin VM, Irvine KD. An O-fucose site in the ligand binding domain inhibits Notch activation. Development 2003;130:6411-6421. [PubMed: 14627724]

17. Ahimou F, Mok LP, Bardot B, Wesley C. The adhesion force of Notch with Delta and the rate of Notch signaling. J Cell Biol 2004;167:1217-1229. [PubMed: 15611340] Atomic force microscopy study of Dros. Notch/Delta interaction.

18. Shimizu K, Chiba S, Saito T, Kumano K, Takahashi T, Hirai H. Manic fringe and lunatic fringe modify different sites of the Notch2 extracellular region, resulting in different signaling modulation. J Biol Chem 2001;276:25753-25758. [PubMed: 11346656]

19. Yang LT, Nichols JT, Yao C, Manilay JO, Robey EA, Weinmaster G. Fringe glycosyltransferases differentially modulate Notch1 proteolysis induced by Delta1 and Jagged1. Mol Biol Cell 2005;16:927-942. [PubMed: 15574878]

20. Rampal R, Li AS, Moloney DJ, Georgiou SA, Luther KB, Nita-Lazar A, Haltiwanger RS. Lunatic fringe, manic fringe, and radical fringe recognize similar specificity determinants in O-fucosylated epidermal growth factor-like repeats. J Biol Chem 2005;280:42454-42463. [PubMed: 16221665]

21. Nichols JT, Miyamoto A, Olsen SL, D’Souza B, Yao C, Weinmaster G. DSL ligand endocytosis physically dissociates Notch1 heterodimers before activating proteolysis can occur. J Cell Biol 2007;176:445-458. [PubMed: 17296795]

22. Ladi E, Nichols JT, Ge W, Miyamoto A, Yao C, Yang LT, Boulter J, Sun YE, Kintner C, Weinmaster G. The divergent DSL ligand D113 does not activate Notch signaling but cell autonomously attenuates signaling induced by other DSL ligands. J Cell Biol 2005;170:983-992. [PubMed: 16144902]

23. Moloney DJ, Shair LH, Lu FM, Xia J, Locke R, Matta KL, Haltiwanger RS. Mammalian Notch1 is modified with two unusual forms of O-linked glycosylation found on epidermal growth factor-like modules. J Biol Chem 2000;275:9604-9611. [PubMed: 10734111]

24. Chen J, Moloney DJ, Stanley P. Fringe modulation of Jagged1-induced Notch signaling requires the action of beta 4galactosyltransferase-1. Proc Natl Acad Sci U S A 2001;98:13716-13721. [PubMed: 11707585]

25. Aoki K, Perlman M, Lim JM, Cantu R, Wells L, Tiemeyer M. Dynamic developmental elaboration of N-linked glycan complexity in the Drosophila melanogaster embryo. J Biol Chem 2007;282:91279142. [PubMed: 17264077]

26. Chen J, Lu L, Shi S, Stanley P. Expression of Notch signaling pathway genes in mouse embryos lacking b4galactosyltransferase-1. Gene Expr Patterns 2006;6:376-382. [PubMed: 16412699]

27. Shao L, Moloney DJ, Haltiwanger R. Fringe modifies O-fucose on mouse Notch1 at epidermal growth factor-like repeats within the ligand-binding site and the Abruptex region. J Biol Chem 2003;278:7775-7782. [PubMed: 12486116]

28. Xu A, Lei L, Irvine KD. Regions of Drosophila notch that contribute to ligand binding and the modulatory influence of fringe. J Biol Chem 2005;280:30158-30165. [PubMed: 15994325]

29. Hambleton S, Valeyev NV, Muranyi A, Knott V, Werner JM, McMichael AJ, Handford PA, Downing AK. Structural and functional properties of the human Notch-1 ligand binding region. Structure 2004;12:2173-2183. [PubMed: 15576031]

30. Rampal R, Arboleda-Velasquez J, Nita-Lazar A, Kosik KS, Haltiwanger RS. Highly conserved Ofucose sites have distinct effects on notch1 function. J Biol Chem 2005;280:32133-32140. [PubMed: 15994302]

31. Shi S, Ge C, Luo Y, Hou X, Haltiwanger RS, Stanley P. The threonine that carries fucose, but not fucose, is required for Cripto to facilitate Nodal signaling. J Biol Chem 2007;282:20133-20141. [PubMed: 17504756]

32. Li Y, Lei L, Irvine KD, Baker NE. Notch activity in neural cells triggered by a mutant allele with altered glycosylation. Development 2003;130:2829-2840. [PubMed: 12756168] 
33. Nita-Lazar A, Haltiwanger RS. Methods for analysis of O-linked modifications on epidermal growth factor-like and thrombospondin type 1 repeats. Methods Enzymol 2006;417:93-111. [PubMed: 17132500]

34. Wang Y, Shao L, Shi S, Harris RJ, Spellman MW, Stanley P, Haltiwanger RS. Modification of epidermal growth factor-like repeats with O-fucose. Molecular cloning and expression of a novel GDP-fucose protein O- fucosyltransferase. J Biol Chem 2001;276:40338-40345. [PubMed: 11524432]

35. Luo Y, Haltiwanger RS. O-fucosylation of notch occurs in the endoplasmic reticulum. J Biol Chem 2005;280:289-294.

36*. Okajima T, Irvine KD. Regulation of Notch Signaling by O-Linked Fucose. Cell 2002;111:893904. [PubMed: 12526814] RNAi-mediated knock down of Dros. OFUT1 gives Fringe-dependent and Fringe-independent Notch signaling defects.

37*. Sasamura T, Sasaki N, Miyashita F, Nakao S, Ishikawa HO, Ito M, Kitagawa M, Harigaya K, Spana E, Bilder D, et al. neurotic, a novel maternal neurogenic gene, encodes an O-fucosyltransferase that is essential for Notch-Delta interactions. Development 2003;130:4785-4795. [PubMed: 12917292] The neurotic mutation inactivates the OFUT1 gene giving the same Notch signaling phenotype as RNAi-mediated knock down.

38*. Shi S, Stanley P. Protein O-fucosyltransferase 1 is an essential component of Notch signaling pathways. Proc Natl Acad Sci U S A 2003;100:5234-5239. [PubMed: 12697902] Targeted inactivation of the mouse Pofut1 gene causes global Notch signaling defects and embryonic death at E9.5.

39. Shi S, Stahl M, Lu L, Stanley P. Canonical Notch signaling is dispensable for early cell fate specifications in mammals. Mol Cell Biol 2005;25:9503-9508. [PubMed: 16227600]

40. Hidalgo A, Ma S, Peired AJ, Weiss LA, Cunningham-Rundles C, Frenette PS. Insights into leukocyte adhesion deficiency type 2 from a novel mutation in the GDP-fucose transporter gene. Blood 2003;101:1705-1712. [PubMed: 12406889]

41. Hellbusch CC, Sperandio M, Frommhold D, Yakubenia S, Wild MK, Popovici D, Vestweber D, Grone HJ, von Figura K, Lubke T, et al. Golgi GDP-fucose transporter-deficient mice mimic congenital disorder of glycosylation IIc/leukocyte adhesion deficiency II. J Biol Chem 2007;282:10762-10772. [PubMed: 17276979]

42. Ishikawa HO, Higashi S, Ayukawa T, Sasamura T, Kitagawa M, Harigaya K, Aoki K, Ishida N, Sanai Y, Matsuno K. Notch deficiency implicated in the pathogenesis of congenital disorder of glycosylation IIc. Proc Natl Acad Sci U S A 2005;102:18532-18537. [PubMed: 16344471]

43. Ishida N, Kawakita M. Molecular physiology and pathology of the nucleotide sugar transporter family (SLC35). Pflugers Arch 2004;447:768-775. [PubMed: 12759756]

44. Smith PL, Myers JT, Rogers CE, Zhou L, Petryniak B, Becker DJ, Homeister JW, Lowe JB. Conditional control of selectin ligand expression and global fucosylation events in mice with a targeted mutation at the FX locus. J Cell Biol 2002;158:801-815. [PubMed: 12186857]

45. Rampal R, Luther KB, Haltiwanger RS. Notch signaling in normal and disease States: possible therapies related to glycosylation. Curr Mol Med 2007;7:427-445. [PubMed: 17584081]

46. Schweisguth F. Notch signaling activity. Curr Biol 2004;14:R129-138. [PubMed: 14986688]

47*. Visan I, Yuan JS, Tan JB, Cretegny K, Guidos CJ. Regulation of intrathymic T-cell development by Lunatic Fringe- Notch1 interactions. Immunol Rev 2006;209:76-94. [PubMed: 16448535] Identifies an important role for mouse Lfng in enhancing $\mathrm{T}$ cell development in the thymus.

48. Sparrow DB, Chapman G, Wouters MA, Whittock NV, Ellard S, Fatkin D, Turnpenny PD, Kusumi K, Sillence D, Dunwoodie SL. Mutation of the LUNATIC FRINGE Gene in Humans Causes Spondylocostal Dysostosis with a Severe Vertebral Phenotype. Am J Hum Genet 2006;78:28-37. [PubMed: 16385447]

49. Hahn KL, Johnson J, Beres BJ, Howard S, Wilson-Rawls J. Lunatic fringe null female mice are infertile due to defects in meiotic maturation. Development 2005;132:817-828. [PubMed: 15659488] Lfng mutant mice may survive to adulthood allowing the identification of a role for Lfng in female meiosis.

50. Thomsen B, Horn P, Panitz F, Bendixen E, Petersen AH, Holm LE, Nielsen VH, Agerholm JS, Arnbjerg J, Bendixen C. A missense mutation in the bovine SLC35A3 gene, encoding a UDP-N- 
acetylglucosamine transporter, causes complex vertebral malformation. Genome Res 2006;16:97105. [PubMed: 16344554]

\section{Abbreviations}

\section{Pofut1}

protein O-fucosyltransferase 1

Lfng

Lunatic Fringe

Mfng

Manic Fringe

Rfng

Radical Fringe

NECD

Notch extracellular domain

NICD

Notch intracelluar domain

N-AP

NECD-alkaline phosphatase

EGF

epidermal growth factor

ER

endoplasmic reticulum

CSL

CBF-1/Suppressor-of-hairless/Lag-1

Maml

Mastermind-like 


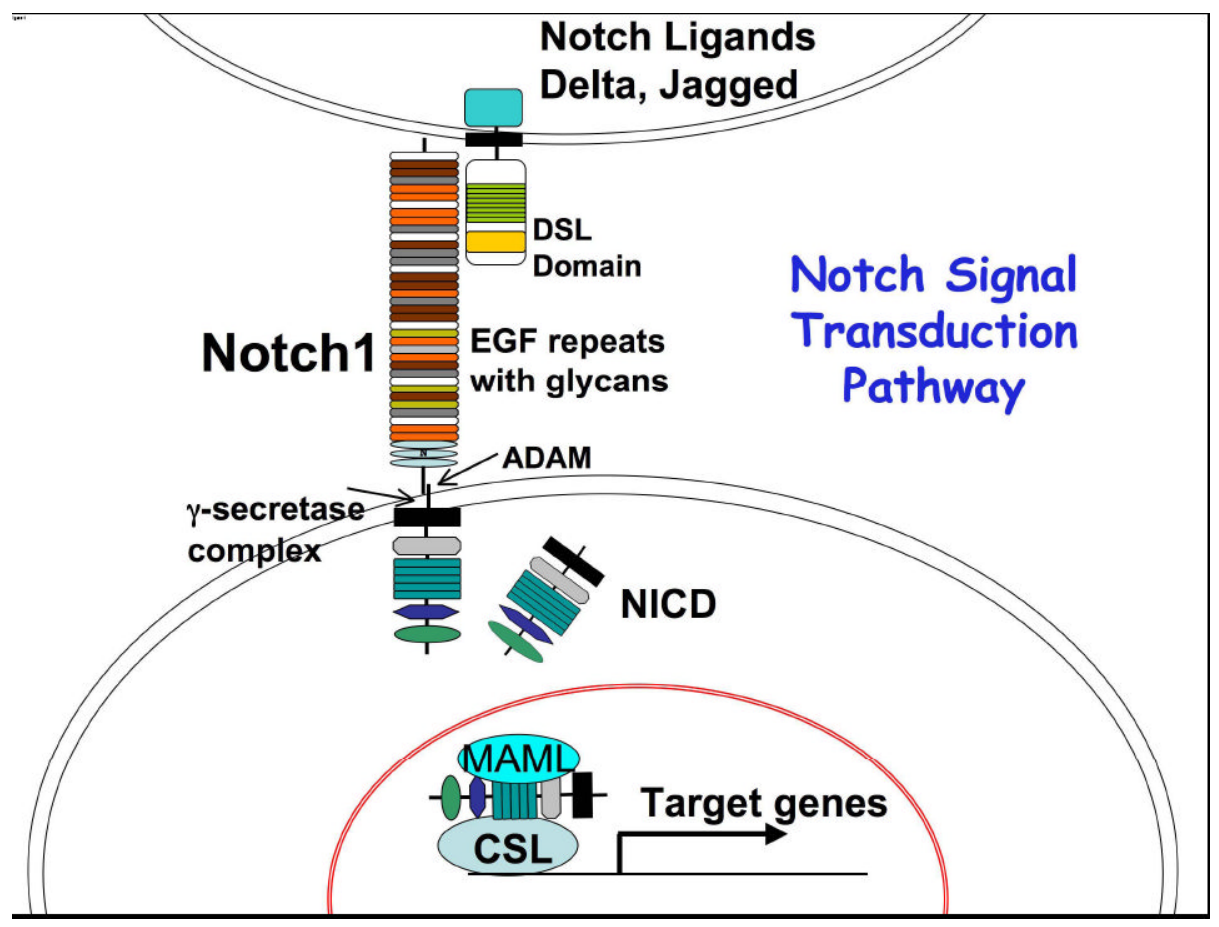

Fig. 1. Canonical Notch signal transduction pathway

Mammalian Notch1 is given as an example of Notch receptors in mammals and Drosophila. Notch receptors are cleaved by furin in the Golgi compartment to form heterodimers that span the plasma membrane once. The EGF repeats of Notch1 are colored according to the O-fucose, O-glucose and complex N-glycans attached as described in Fig. 2. Notch ligands also carry EGF repeats but to date no functional effects have been observed in the absence of O-fucose glycans. Ligands require their N-terminal DSL (Delta/Serrate/Lag-2) domain to bind to Notch receptors. Ligand binding is followed by endocytosis of NECD into the ligand cell, cleavage by an ADAM protease at a juxtamembrane site followed by cleavage by a complex containing $\gamma$-secretase in the transmembrane region (leaving Val $1744 \mathrm{~N}$-terminal in the case of Notch1). Released NICD goes to the nucleus to complex with the CSL transcriptional repressor bound to the consensus GCTGATAG in promoter regions of genes. The co-activator MAML is recruited and target genes are activated. Well characterized Notch target genes include the Hes and Hey transcriptional repressors. 


\section{Glycans of Mammalian Notch1 EGF Repeats}

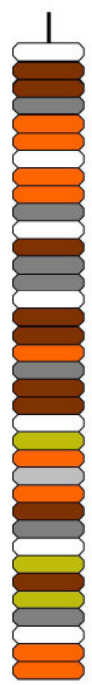

O-Fucose glycan

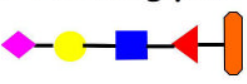

O-Glucose glycan

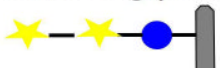

Complex $\mathrm{N}$-glycan

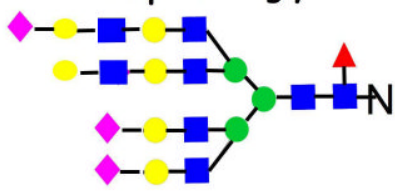

No glycan

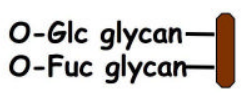

O-Fuc glycan-

$\mathrm{N}$ - glycan-

O-Fuc glycan-

$\mathrm{N}$ - glycan-

- GICNAC

- Man

- Fuc

Sialic acid

- Glucose

Notch1

EGF repeats

Fig. 2. The Glycans of Mouse Notch1

Notch1 receptor EGF repeats have consensus sites for $O$-fucose $\left(\mathrm{C}^{2} \mathrm{X}_{4-5} \mathrm{~S} / \mathrm{TC}^{3}\right)$ [27] and $O$ glucose $\left(\mathrm{C}^{1} \mathrm{XSXPC}{ }^{2}\right)$ glycans [23] while $N$-glycans are added at certain AsnXSer/Thr consensus sequons. Structural analyses have been performed on Notch1 synthesized as ECD fragments in mammalian cells. The largest $\mathrm{O}$-fucose glycan observed to date is sialic acid $\alpha$ $(2,3) \mathrm{Gal} \beta(1,4) \mathrm{GlcNAc} \beta(1,3) \mathrm{Fuc}-\mathrm{O}$ and the largest O-glucose glycan is Xyl $\alpha(1,3) \mathrm{Xyl} \alpha(1,3) \mathrm{O}$ Glc-O. O-glycans initiated by O-GalNAc on Ser/Thr may also be present but have not been reported. 
Table 1

In vivo consequences of modifying O-fucose glycans

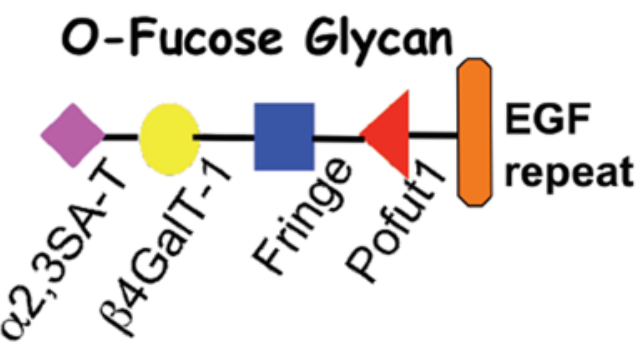

\begin{tabular}{|l|l|l|l|}
\hline $\begin{array}{l}\text { Activity } \\
\text { Eliminated } \\
\text { (Dros./Mamm.) }\end{array}$ & Missing sugar & Drosophila & Mammals \\
\hline OFUT1/Pofut1 & Fucose & Larval death & Death at mid-gestation \\
\hline GDP-Fucose transporter & Fucose & ts Notch defective phenotypes & Leukocyte extravasation phenotype \\
\hline GDP-Fucose synthesis & Fucose & Notch signaling at boundary of wing disc & No obvious Notch phenotype \\
\hline FNG/Lfng & GlcNAc & Boundaries wing. eve, leg & Somitogenesis (severe) Block in female meiosis \\
\hline UDP-GlcNAc transporter & GlcNAc & Fringe-related defects & Somitogenesis (severe) \\
\hline B4GalT-1 & Galactose & ? & Somitogenesis (mild) \\
\hline
\end{tabular}

Genetic strategies to eliminate various glycosylation activities required for O-fucose glycan synthesis have been used [5,10]. The transfer of O-fucose to Notch receptors is catalysed by Pofut [34] and not Pofut2 [31]. Pofut1 and the Drosophila ortholog OFUT1 reside in the ER [13,35]. The nucleotide sugar GDP-Fucose donor is synthesized in the cytoplasm and transported into the secretory pathway by a GDP-Fucose transporter. Inactivation of mouse Pofut1 or Drosophila OFUT1 leads to severe Notch signaling defects and embryonic death [36-38]. The phenotypes are cell-autonomous and highly characteristic of disrupted Notch signaling. Surprisingly, removal of Pofut1 in the 2-cell blastocyst has no consequences prior to gastrulation [39]. Interestingly, inactivation of the Golgi GDP-Fucose transporter is associated with leukocyte adhesion defects in humans [40] and in mice [41], and gives a mild, temperature-sensitive Notch phenotype in flies [42]. Transport of GDP-Fucose may also be mediated by another transporter [43]. Inhibition of GDP-Fucose synthesis leads to a subset of Notch signaling defects in Drosophila [13-15] but no visible Notch phenotype in mice [44], potentially because of maternal sources of GDP-Fucose. Clearly none of these strategies uniquely targets Notch receptors since a number of proteins have EGF repeats with the consensus recognized by OFUT1/Pofut1 [45], and all glycoproteins acted on by any fucosyltransferase will be affected by the loss of GDP-Fucose or the GDP-Fucose transporter. In addition, elimination of a glycosyltransferase may have pleiotrophic consequences if it has more than one function, or it acts in both a cell-autonomous and non cell-autonomous fashion [13-15]. Overexpression artefacts are a concern when transgenes are expressed under the control of exogenous promoters and/or in an ectopic location, since regulated Notch signaling is notoriously sensitive to the level of Notch receptor activity [46,47]. Drosophila has one Fringe while mammals have three (Lfng, Mfng and Rfng). Mutations of Fringe give Notch signaling defects that manifest at developmental boundaries in the formation of wings, legs and eyes in Drosophila [7]. In mammals, including humans [48], Lfng is required for Notch signaling during somitogenesis, and affects female meiosis [49] and T cell development in mice [47]. In vivo functions of Rfng are not noticeably developmental [10] and Mfng mutant mice have not been described. Fringe connection encodes a transporter that transports UDP-GlcNAc and mutants have Fringe-related defects in Drosophila [7]. A UDP-GlcNAc transporter mutation is also responsible for skeletal defects in cattle [50]. In CHO cells, $\beta 4$ GalT-1 adds Gal to O-fucose glycans [24] and $\beta 4$ GalT-1 null mice have mild Notch signaling defects [26]. In CHO cells O-fucose glycans receive a 2,3-linked sialic acid [23,24]. There is no evidence for Notch signaling defects in sialyltransferase knockout mice [5], but they may have a mild phenotype that is not readily apparent. O-fucose, red triangle; GlcNAc, blue square; Gal, yellow circle; sialic acid, purple diamond. 